\title{
Temperature Robust PCA Based Stress Monitoring Approach
}

\author{
Jabid Quiroga1, a , John Quiroga ${ }^{2, b}$, Luis Mujica ${ }^{3, c}$, Rodolfo Villamizar ${ }^{1, d}$, \\ Magda Ruiz ${ }^{3, \mathrm{e}}$ \\ ${ }^{1}$ Universidad Industrial de Santander, Cra. 27 Calle 9, Bucaramanga, Colombia \\ ${ }^{2}$ Universidad Santo Tomas, Cra 18 Calle 9, Bucaramanga, Colombia \\ ${ }^{3}$ Department of Mathematics, EEBest UPC Campus Diagonal-Besós, Barcelona, Spain.

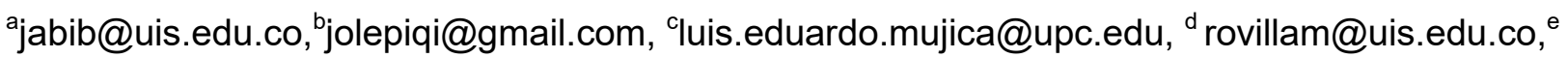 \\ magda.ruiz@upc.edu
}

Keywords: Stress monitoring, Guided waves, PCA, Temperature robust.

\begin{abstract}
In this paper, a guided wave temperature robust PCA-based stress monitoring methodology is proposed. It is based on the analysis of the longitudinal guided wave propagating along the path under stress. Slight changes in the wave are detected by means of PCA via statistical $\mathrm{T}^{2}$ and $\mathrm{Q}$ indices. Experimental and numerical simulations of the guided wave propagating in material under different temperatures have shown significant variations in the amplitude and the velocity of the wave. This condition can jeopardize the discrimination of the different stress scenarios detected by the PCA indices. Thus, it is proposed a methodology based on an extended knowledge base, composed by a PCA statistical model for different discrete temperatures to produce a robust classification of stress states under variable environmental conditions. Experimental results have shown a good agreement between the predicted scenarios and the real ones.
\end{abstract}

\section{INTRODUCTION}

Stresses in structures have great influence in the performance during the operation, affecting its strength, expected operational life and dimensional stability. A suitable alternative is the use of lowfrequency ultrasonic guided waves. The ultrasonic guided waves have the ability to propagate along relative long distances, compared to most bulk wave NDE methods, while still maintaining sensitivity to condition changes in the structure [1]. Guided waves are used for damage detection, damage localization and material characterization. [2-7].

Guided waves propagating in materials under stress conditions present some changes in the wave pattern with respect to the pattern for different stresses, which can be monitored under two approaches. The first one is based on acoustoelasticity, which involves the evaluation of the ultrasonic bulk wave velocities. However, the acoustoelasticity in a specific material is difficult to generalize. In addition, the acoustoelastic effect is small for metals, typically about $0.001 \%$ per MPa of applied stress [8]. The second one relies on particular features in the waveform attributed to the guided wave propagation in a medium under stress. These changes can be traced by using statistics tools, such as PCA. In this paper, a temperature robust alternative approach is being considered by using a PCA statistical data driven model of the guided wave pattern.

Stress effect over the propagation of guided waves. The presence of external mechanical stresses in a material causes modification of its structural morphology and its mechanical and acoustic behavior. Then, the propagation of the guided waves is modified in terms of velocity and attenuation. Under stress, these quantities depend on the direction and polarization of the waves as well as on the direction of the applied stress [1]. The bulk wave velocity variations as a function of the stress applied to the propagating medium is governed by the acoustoelasticity effect. The acoustoelasticity effect is founded on the nonlinear elasticity theory. This new model includes three acoustoelastic constants $(l, m, n)$, in addition to the Lamé constants ( $\lambda$ and $\mu)$ [2]. A first order 
approximation of the bulk longitudinal wave velocity for an isotropic material subject to uniaxial stress is presented in Eq. 1 [3].

$$
v_{\sigma}=\sqrt{\frac{\lambda+2 \mu}{\rho}}\left\{1+\frac{\sigma}{2(\lambda+2 \mu)(3 \lambda+2 \mu)}\left(\frac{\lambda+\mu}{\mu}(4 \lambda+10 \mu+4 m)+\lambda+2 l\right)\right\},
$$

where $\sigma$ is the uniaxial stress, $\rho$ is the mass density, and $\boldsymbol{v}_{\mathbf{0}}=\sqrt{(\boldsymbol{\lambda}+2 \boldsymbol{\mu}) / \boldsymbol{\rho}}$, is the bulk longitudinal wave velocity in a stress free medium. The expression inside of the curly brackets governs the velocity factor attributed to the stress, which is generally very small for most of metallic materials. Therefore, velocity of the ultrasonic waves is not significantly altered by the applied stresses becoming difficult to determine the velocity variation. Although, the acoustoelasticity predicts the change of ultrasonic bulk velocities, some recent works have shown their extensions to the guided waves [1][3].

On the other hand, experimentally, the presence of stress produces changes in the wave pattern, in time domain. It is manifested by the arrival time of the wave, which can be mainly endorsed to a change in the length of the propagation path attributed to the elasticity, and changes in the propagation velocity (acoustoelasticity). The combination of both effects can be appreciated in a time delay when guided waves of different stresses conditions are compared. This slightly variations in the wave pattern can be revealed using a statistical model PCA based.

Temperature effect over the propagation of guided waves. There are many effects of temperature fluctuation in the guided wave pattern. The first effect and probably the most noticeable is the amplitude variation. In general, it is reported a magnitude reduction when the temperature is increasing [4]. In addition to the amplitude reduction is also found a velocity decrement (increase time of arrival) as the temperature is raising [5]. Temperature varying conditions in the system substantially alter the recorded waveform and its effects can jeopardize any intent to detect variations of the structure condition. In some conditions, the temperature varying effect in the wave can be relevant and influences directly the monitoring result [6]. Thus, temperature robustness is an obligate requirement of practical applications SHM schemes.

PCA. Principal component analysis (PCA) has been used in Structural Health Monitoring (SHM), for extracting structural damage features and to discriminate features from damaged and undamaged structures [7][8][4][9]. In SHM PCA-based, data is arranged in a matrix $\mathbf{X}$ as follows in Eq. 2

$$
\mathrm{X}=\left[\begin{array}{cccc}
x_{11} & x_{12} & \ldots & x_{1 j} \\
\ldots & \ldots & \ldots & \ldots \\
x_{i 1} & x_{i 2} & \ldots & x_{i j} \\
\ldots & \ldots & \ldots & \ldots \\
x_{n i} & x_{n 2} & \ldots & x_{n j}
\end{array}\right]=\left[\vartheta_{1}\left|\vartheta_{2}\right| \ldots\left|\vartheta_{j}\right| \ldots \vartheta_{n}\right]
$$

This $\mathbf{X}$ matrix contains information from the measurement $j$ and $n$ experimental trials. Each row vector $x_{i}$ represents measurements from the sensors attached to the structure for a specific experiment trial. In the same way, each column vector $v_{i}$ represents samples from sensors (one variable) in the whole set of experiment trials.

Two statistics scores associated to the PCA are $T^{2}$ and $Q$ statistics. The first one is based on analyzing the residual data matrix to represent the variability of the data projection in the residual subspace. In other words, $T^{2}$-statistic is a measure of the variation of each sample within the PCA model. Besides, $Q$-statistic denotes the change of the events that are not explained by the model of principal components. It is a measure of the difference, or residual between a sample and its projection into the model. Normally, $Q$-statistic is much more sensitive than $T^{2}$-statistic. This is because $Q$ is very small and therefore any minor change in the system characteristics will be observable. $T^{2}$ has great variance and therefore requires a great change in the system characteristic to be detectable. 


\section{EXPERIMENTAL SETUP}

The test bench used in this research represents a scaled mimic of an actual installation. A 1" $6 \mathrm{~m}$ of length, Sch. 40 A-106 pipeline supported at the free ends by fixed support is used and a variable load is applied on the half of the length emulating the stress produced for a fault in the foundation. The different stress conditions are either produced by changing the magnitude of the reaction in the variable support located in the middle of the pipeline $(\mathrm{L} / 2)$ or by adding a concentrated force at the middle part of the pipeline. A 2208 Picoscope ${ }^{\circledR}$ is used for signal generation (Gaussian pulse signal of nine cycles) and acquisition of the guided wave. The actuator and the sensor are located at $0.5 \mathrm{~m}$ equidistant from the middle, aligned with the pipe axis. A Matlab ${ }^{\circledR}$ script is implemented to perform processing of this signal. The baseline (nominal condition) is determined considering the absence of deflection in the middle part of the pipe. Now, the magnitude of the variable support is decreasing while the pipeline deflection is increasing in steps of $0.01 \mathrm{~m}$ down of the original axis position (baseline). Every $0.01 \mathrm{~m}$, deflection constitutes a different stress scenario, denominated D1 ( $5 \%$ of the yield strength) up to D4 $(37.5 \%$ of the yield strength), where D1 $=-72.88 \mathrm{kPa}, \mathrm{D} 2=10.31$ $\mathrm{MPa}, \mathrm{D} 3=20.96 \mathrm{MPa}$ and $\mathrm{D} 4=31.62 \mathrm{MPa}$, stresses in the middle of the pipe. The temperature varying conditions in the pipe are attained by irradiating it with infrared lamps.

\section{ROBUST Stress monitoring PCA based approach}

In this work the proposed scheme provides information about changes in the stress level but not the value of stress in itself. Information of the wave propagating along pipeline about its nominal stage is statistically processed by means of PCA, in order to obtain a statistical baseline model represented by the principal components. The stress conditions diagnosis stage is executed by projecting the captured data onto the baseline model, scores, $T^{2}$, and $Q$-Statistics indices are computed to distinguish a new stress condition regard to the baseline case.vThe challenge for the proposed methodology, in a temperature-varying environment, is to discriminate between temperature variations and changes in stress because both produce the same signature in the wave, i.e. changes in amplitude and velocity, which in the studied cases are more pronounced for temperature variations.

A general scheme of the proposed methodology is shown in Fig. 1. To reduce the dominant effect on the wave caused for changing temperature an autocorrelation and normalization is applied to the captured signal. The $\mathbf{X}$ matrix is assembled using the normalized cross-correlation between the actuated and sensed signals. To improve the signal-to-noise ratio, multiple sets of raw signals are captured. A number of 100 experiments have been performed and recorded for each scenario of stress condition. A extended baseline, which contains the wave pattern for a discrete range of temperatures between $24^{\circ} \mathrm{C}$ and $38^{\circ} \mathrm{C}$ is obtained. The principal components (the first 60) are determined using the data of the extended baseline. In the diagnosis stage, the $\mathbf{X}$ matrix for the current state signal is projected onto the PCA. Projections onto some of the first components are obtained and the stress indices $\left(T^{2}\right.$-statistic and $Q$-statistic) are calculated and compared with the baseline values.

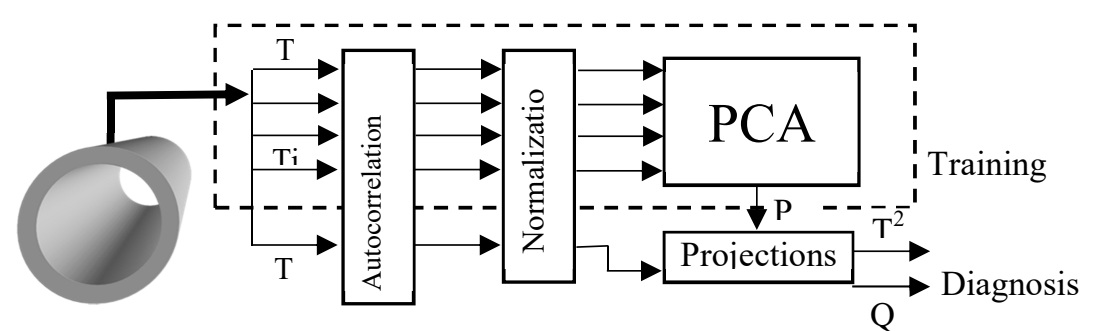

Figure 1. Schematic representation of the proposed robust temperature stress monitoring methodology. 


\section{Analysis of Results}

In Fig. 2, it is shown the result for the different stressed scenarios at different surrounding temperatures. The results are obtained using the proposed scheme. Comparing the statistical index clusters for different temperatures at different stresses scenarios in Fig. 2, it can be noted, they are grouping around a defined region. A substantial improvement is appreciated with respect to the scheme without the preprocessing and the extended knowledge base.

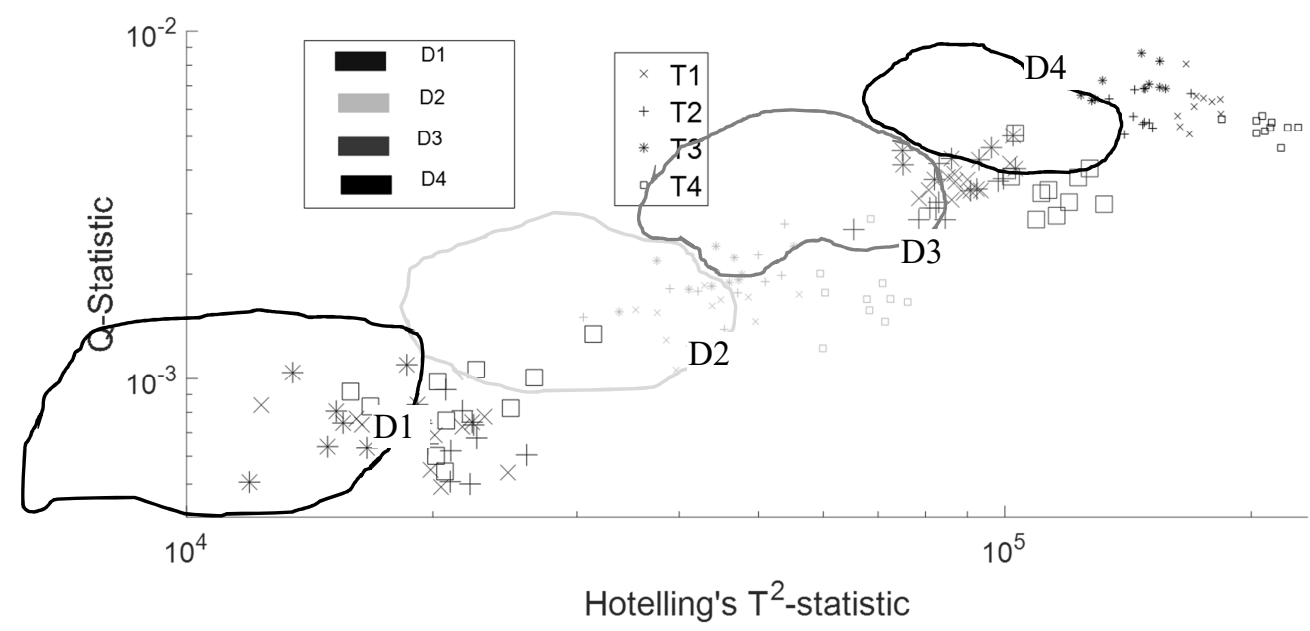

Figure 2. Statistical PCA índices at different stress conditions and at different room temperatures a). at $28^{\circ} \mathrm{C}$, b)at $30^{\circ} \mathrm{C}$, c)at $33^{\circ} \mathrm{C}$ and d)at $38^{\circ} \mathrm{C}$.

\section{Conclusions}

Based on the experimental results, we can conclude that the proposed scheme is effective unmasking the temperature effect in the statistical indices used in the monitoring. Some slightly variations are presented in the diagnosis stage under different surroundings temperatures. In order to assure an appropriate monitoring, the same piezo-actuation (PZT couple) must be maintained in the range of temperatures used in the setup stage. The guided wave pattern is highly dependent of the PZT parameters and the characteristics of the adhesive layer. Because the methodology is based on guided wave pattern recognition particular attention must be considered in subjects such as PZT aging, condition of the bounding layer, which are not covered in this paper.

\section{Acknowledgment}

This work has been partially funded by the Spanish Ministry of Economy and Competitiveness through the research project DPI2014-58427-C2-1-R, and by the Catalonia Government through the research project 2014 SGR 859.

\section{References}

[1] S. Chaki and G. Bourse: Stress level measurement in prestressed steel strands using acoustoelastic effect. Exp. Mech., vol. 49, no. 5 (2009), pp. 673-681.

[2] D.S.Hughes, J.L.Kelly: Second-Order Elastic Deformations of Solids. Phys. Rev., vol. 92, no. 5, (1953) pp. 1145-1150,.

[3] S. Chaki and G. Bourse: Guided ultrasonic waves for non-destructive monitoring of the stress levels in prestressed steel strands. Ultrasonics, vol. 49, no. 2, (2009) pp. 162-171.

[4] F. Gharibnezhad: Robust Damage Detection in Smart Structures. PhD Thesis. U.P. Cataluña (2014). 
[5] J. P. Andrews: Lamb Wave Propagation in Varying Thermal Enviroments. Air Force Institute of Technology Air, 2007.

[6] A. J. Croxford, P. D. Wilcox, B. W. Drinkwater, and G. Konstantinidis; Strategies for guidedwave structural health monitoring. Proc. R. Soc. A Math. Phys. Eng. Sci., vol. 463, no. 2087, (2007) pp. 2961-2981

[7] L. Zhong, H. Song, and B. Han; Extracting structural damage features: Comparison between PCA and ICA. Lect. Notes Control Inf. Sci., vol. 345, (2006), pp. 840-845.

[8] A. M. Yan, G. Kerschen, P. De Boe, and J. C. Golinval: Structural damage diagnosis under varying environmental conditions - Part I: A linear analysis. Mech. Syst. Signal Process., vol. 19, no. 4,(2005), pp. 847-864.

[9] A. Hot, G. Kerschen, E. Foltête, and S. Cogan: Detection and quantification of non-linear structural behavior using principal component analysis. Mech. Syst. Signal Process., vol. 26, no. 1, (2012), pp. 104-116. 